\title{
Practical Emergency Ultrasound Flashcards with Augmented Reality in Teaching Point-of-Care Ultrasound in ER
}

\author{
Kamonwon lenghong, Praew Kotruchin, Thanat Tangpaisarn, Korakot Apiratwarakul* \\ Department of Emergency Medicine, Faculty of Medicine, Khon Kaen University, Khon Kaen, Thailand
}

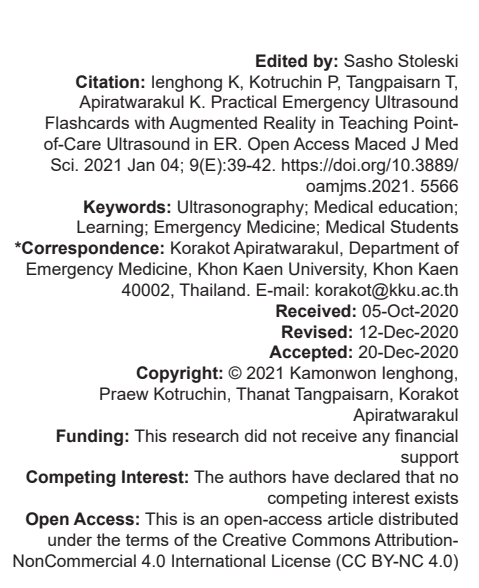

Introduction

In the emergency department, point-of-care ultrasound (POCUS) is widely accepted as an essential procedure, which can aid clinicians in diagnosing and in performing resuscitation procedures. In addition, there is evidence that it can be performed effectively by nonspecialist physicians after they have received limited training [1], [2], [3], [4]. Therefore, POCUS training is recommended for medical students, for emergency medicine residents, and for other subspecialty physicians worldwide.

At present, ultrasound has been implemented in the medical curricula of many medical schools [5], [6], [7], [8]. However, the study of the POCUS learning in the medical curricula in Thailand is still limited given that the materials for learning POCUS in Thailand are mostly in the form of textbooks, which cannot show the visual animation of the ultrasound clips. Hence, this factor makes it difficult for students to recognize and to understand the various characteristics of ultrasound.

The novice learning tool, which was named "The Practical Ultrasound Flashcards with Augmented Reality," was developed by members of the Faculty of
Emergency Medicine at Khon Kaen University in 2019. This POCUS learning tool aims to give the medical students easy access to POCUS knowledge and to increase their enjoyment of learning.

In terms of the POCUS learning for medical students, a 4-week POCUS training was conducted with the $6^{\text {th }}$ year medical students at Khon Kaen University during their rotation in emergency medicine, which included didactic lectures as well as bedside ultrasound. The primary objective in this study was to evaluate the POCUS knowledge of the $6^{\text {th }}$ year medical students before and after their rotations in emergency medicine. This process was carried out by comparing the two methods of the learning process: The traditional training and the traditional training, which included the self-study with "The Practical Ultrasound Flashcards with Augmented Reality."

\section{Methods}

\section{Study design}

This randomized controlled trial was conducted in a tertiary university hospital in Thailand. Ethics 
approval was provided by the Khon Kaen University Ethics Committee for Human Research, and the approval was registered with the Thai Clinical Trials Registry (HE631197).

\section{Participants}

The participants were the $6^{\text {th }}$ year medical students who were studying in the emergency medicine during their rotation at the Department of Emergency Medicine at Srinagarind Hospital in Khon Kaen. Sample random sampling was used to enroll the students in this study. The written informed consent was obtained from each participant before enrollment.

\section{Sample size}

The sample size for the analysis of the estimated sample size for two samples with repeated measures was determined. We hypothesized that the difference in knowledge between the two groups would be approximately $5 \%$ and that there would be a moderate degree of consistency between the pre-test scores and the post-test scores. The power analysis was determined using an alpha of 0.05 and a power of 0.80 . This resulted in an estimated desired effect sample size of at least 23 subjects in each group. The medical students, who were not participating in this particular rotation, were excluded.

\section{Study protocol}

Before any intervention, the students' baseline POCUS knowledge was tested using a multiple-choice pre-test. Students were divided into two groups using sample random sampling: The first group of students only received the traditional POCUS learning (the control group), while the second group of students received the traditional POCUS learning, as well as the ultrasound flashcards (the flashcard group). After this, students in both groups attended the traditional POCUS training course, which was provided by staff members of the department of emergency medicine, who are specialists in POCUS.

The traditional POCUS training course consisted of the 11 didactic lectures (ocular, lung, abdominal aorta, gallbladder, small bowel, appendix, kidney, skin and soft tissue, deep venous thrombosis, ultrasound protocols, and cardiology) and bedside ultrasound with the real patients (three sessions per week at $3 \mathrm{~h}$ per session).

During the 4 weeks of the emergency ultrasound rotation, the students in the flashcard group were allowed to learn POCUS from the ultrasound flashcards as often as they wished. After the training period, both of the student groups were evaluated using a multiple-choice post-test.
The pre- and post-test examination was consisted of 20 POCUS questions with five multiple choice. The scoring system was 1 point per each 1 POCUS questions. Thus, the minimum score and maximum score were 0 and 20 points, respectively.

After the rotation, the students in the flashcard group were asked to a complete self-assessment questionnaire with a Likert scale ranging from 1 to 5 to rate their attitudes and perceptions about the flashcards. Moreover, open comments were used to assess any problems that the students might have had while using the flashcards as a learning tool.

\section{The description of the flashcards}

The practical ultrasound flashcards with augmented reality were developed by Kamonwon lenghong and Praew Kotruchin who are both members of the faculty. The set was designed in the form of 125 pages of cards that contain 20 ultrasound clips and 11 aspects of POCUS knowledge - all of which are included in all of the didactic lectures in the traditional POCUS training course. In terms of the technology of augmented reality, the users were able to download the application on their android phones and to play the animation of ultrasound clips in real time. (Figure 1).

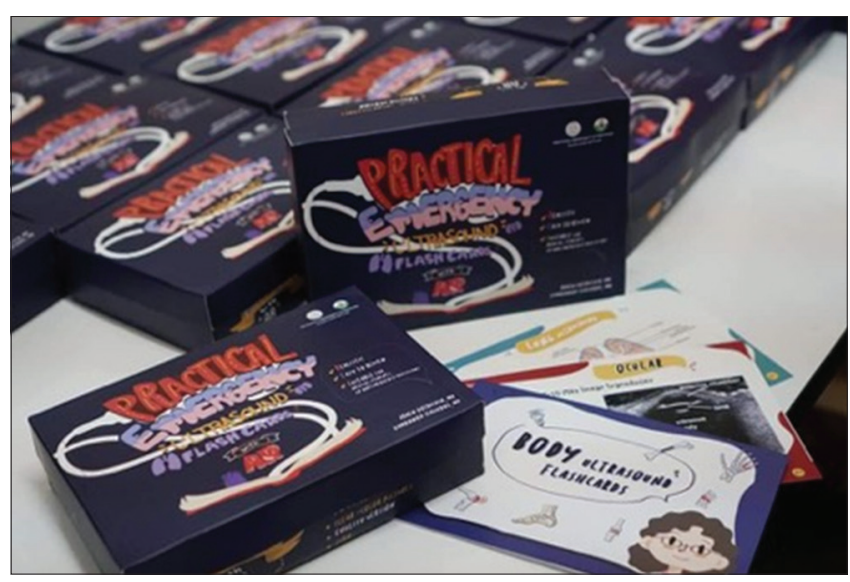

Figure 1: New learning tool named "The Practical Ultrasound Flashcard with AR"

\section{Statistical analysis}

Categorical variables were expressed as frequencies and percentages, while the continuous data were expressed as means and standard deviations. In terms of the baseline characteristics, the differences between groups were compared using an independent sample t-test. An analysis of the covariance model was used to compare the post-test scores of the two groups and to adjust the baseline score measurements. Differences in the pre-training tests and the posttraining tests were compared using an independent sample t-test. A two-tailed $p<0.05$ was considered 
to be statistically significant. All data analyses were performed using Stata version 10 (StataCorp, College Station, TX).

\section{Results}

Our program was conducted from May 2020 to June 2020. All of the $6^{\text {th }}$ year medical students, numbering 46 in total, were recruited from the emergency medicine rotation and were randomly assigned to be either in the flashcard group $(n=23)$ or in the control group $(n=23)$. All students completed the trial and were included in the data analysis. The demographic data of the participants are shown in Table 1.

Table 1: The demographics of the participants

\begin{tabular}{llll}
\hline Characteristics & The flashcard group & The control group & p-value \\
\hline Age (mean) & 22.61 & 22.09 & 0.081 \\
Male, $\mathrm{n}$ & 11 & 12 & 0.774 \\
\hline
\end{tabular}

It was found that the students in the flashcard group had performed better on the POCUS knowledge post-test than those students in the control group. Score improvement in the flashcard group was higher than another group with statistically significant $(p<0.001)$ (Table 2). Most of the students had been satisfied with the flashcards (mean Likert scores 4.48). In terms of the students' degrees of confidence to perform POCUS after using the flashcards, the students had rated their mean confidence score at 3.96 out of 5.0. In terms of the open comments, most students indicated that the flashcards had helped them to better enjoy the process of learning POCUS.

Table 2: The comparison of mean scores of pre- and post-test between the flashcard and control groups

\begin{tabular}{llll}
\hline Characteristics, mean (SD) & The flashcard group & The control group & p-value \\
\hline Pre-test score & $10.30(2.63)$ & $10.83(2.31)$ & 0.479 \\
Post-test score & $17.65(1.85)$ & $16.43(2.43)$ & 0.062 \\
Score improvement & 7.35 & 5.6 & $<0.001$ \\
(post-test vs. pre-test) & & &
\end{tabular}

\section{Discussion}

This is the first study to evaluate the new POCUS learning tool with the $6^{\text {th }}$ year medical students using the randomized prospective method. From the results of the multiple-choice test, we found that the participants had significantly improved. This may suggest that the flashcards, which were developed, had been successful in improving the students' basic POCUS knowledge. It was shown that the average score had improved from 5.6 to 7.35 , which is equivalent to $8.75 \%$. The two groups of students had the basic POCUS knowledge training and the second group had an extra material to learn from (flash cards); therefore, it may be expected that those who have more material to study from will have better response. The previous studies [9], [10], [11], [12], which had examined the teaching of ultrasound courses for medical students and residents, showed that the knowledge scores had increased after learning. However, it is important to note that the learning tools utilized in those studies were not similar to the one used in this study.

Ultrasound education can be delivered through classic methods, such as didactic presentations or practical hands-on courses. Lectures are often appropriate for teaching the fundamental principles of ultrasound. However, lectures alone cannot replace the essential hands-on training that is critical for obtaining the visuospatial and visuomotor skills, which are necessary for handling a transducer and acquiring images [13], [14]. Hence, the confidence score to perform ultrasound in this study was rated as 3.96 , which means that this new learning tool may not be replacing the skill of hands-on teaching.

It is critical to inspire students while they are learning ultrasound. One of the most fascinating teaching methods, and often the best received, is the ability to connect the clinical data and the represented anatomy/pathophysiology to the ultrasound image in real time [13]. Therefore, the new learning tool in this study can serve to address this issue. Moreover, it received high scores when the participants rated their degrees of satisfaction. In the open comments, the students noted that they had also enjoyed the technology of augmented reality, which had made it easy for them to play the ultrasound clips and to review the POCUS knowledge.

At present, POCUS self-learning can be carried out in many ways, such as the SONOSIM ultrasound solution, which is an online education course [15]. The students can explore various ultrasound pathologies in real time, and they are able to practice the clinical decision-making processes in the ultrasound case scenarios. However, the costs make it expensive, which means that for most students or institutions, this learning tool cannot be easily accessed. Therefore, given that the cost is only $\$ 12.50$ or 400 baht, these flashcards may have a definite advantage over other tools because they are cheaper.

\section{Limitations}

However, our study also had some limitations [16], [17], [18], [19]. First, this was a singlecenter study, which naturally limits the generalizability of the results. Second, the students were evaluated based on their knowledge scores, which are imperfect indicators when evaluating ultrasound skills and clinical decision-making processes based on real scenarios. 


\section{Conclusions}

The findings of this study revealed that the $6^{\text {th }}$ year medical students can effectively learn the POCUS using the ultrasound flashcards. Future studies are needed to further evaluate the POCUS skills and the clinical decision-making processes.

\section{Acknowledgments}

The present study received funding from the Khon Kaen University Faculty of Medicine (IN63257). The authors would like to thank all of the participants, as well as the Department of Emergency Medicine at Khon Kaen University. Thanks to Fred Burton Setzler for acting as English consultant.

\section{References}

1. Blois B. Office-based ultrasound screening for abdominal aortic aneurysm. Can Fam Physician. 2012;58(3):e172-8. PMid:22518906

2. Crisp JG, Lovato LM, Jang TB. Compression ultrasonography of the lower extremity with portable vascular ultrasonography can accurately detect deep venous thrombosis in the emergency department. Ann Emerg Med. 2010;56(6):601-10. https://doi. org/10.1016/j.annemergmed.2010.07.010

PMid:20864215

3. Mjølstad OC, Snare SR, Folkvord L, Helland F, Grimsmo A, Torp $\mathrm{H}$, et al. Assessment of left ventricular function by GPs using pocket-sized ultrasound. Fam Pract. 2012;29(5):534-40. https://doi.org/10.1093/fampra/cms009

PMid:22333323

4. Bornemann $P$, Johnson J, Tiglao S, Moghul A, Swain S, Bornemann G, et al. Assessment of primary care physicians' use of a pocket ultrasound device to measure left ventricular mass in patients with hypertension. J Am Board Fam Med. 2015;28(6):706-12. https://doi.org/10.3122/ jabfm.2015.06.140314

PMid:26546645

5. Dinh VA, Frederick J, Bartos R, Shankel TM, Werner L. Effects of ultrasound implementation on physical examination learning and teaching during the first year of medical education. $J$ Ultrasound Med. 2015;34(1):43-50. https://doi.org/10.7863/ ultra.34.1.43

PMid:25542938

6. Dinh VA, Fu JY, Lu S, Chiem A, Fox JC, Blaivas M. Integration of ultrasound in medical education at United States medical schools: A national survey of directors' experiences. J Ultrasound Med. 2016;35(2):413-9. https://doi.org/10.7863/ultra.15.05073 PMid:26782166

7. Hoppmann RA, Rao VV, Bell F, Poston MB, Howe DB, Riffle S, et al. The evolution of an integrated ultrasound curriculum (iUSC) for medical students: 9-year experience. Crit Ultrasound J. 2015;7(1):18.

\section{PMid:26589313.}

8. Blackstock U, Munson J, Szyld D. Bedside ultrasound curriculum for medical students: Report of a blended learning curriculum implementation and validation. J Clin Ultrasound. 2015;43(3):139-44. https://doi.org/10.1002/jcu.22224 PMid:25123564

9. Lee JB, Tse C, Keown T, Louthan M, Gabriel C, Anshus A, et al. Evaluation of a point of care ultrasound curriculum for Indonesian physicians taught by first-year medical students. World J Emerg Med. 2017;8(4):281-6. https://doi.org/10.5847/ wjem.j.1920-8642.2017.04.006 PMid:29123606

10. Greenstein $\mathrm{YY}$, Littauer $\mathrm{R}$, Narasimhan $\mathrm{M}$, Mayo $\mathrm{PH}$ Koenig SJ. Effectiveness of a critical care ultrasonography course. Chest. 2017;151(1):34-40. https://doi.org/10.1016/j. chest.2016.08.1465

PMid:27645689

11. Yamada T, Minami T, Soni NJ, Hiraoka E, Takahashi H, Okubo T, et al. Skills acquisition for novice learners after a point-of-care ultrasound course: Does clinical rank matter? BMC Med Educ. 2018;18(1):202. https://doi.org/10.1186/s12909-018-1310-3 PMid:30134975

12. Dietrich CF, Hoffmann B, Abramowicz J, Badea R, Braden B, CantisaniV, etal. Medical studentultrasound education:AWFUMB position paper, Part I. Ultrasound Med Biol. 2019;45(2):271-81. https://doi.org/10.1016/j.ultrasmedbio.2019.02.020 PMid:30497768

13. Phungoen P, Promto S, Chanthawatthanarak S, Maneepong S, Apiratwarakul K, Kotruchin $\mathrm{P}$, et al. Precourse preparation using a serious smartphone game on advanced life support knowledge and skills: Randomized controlled trial. J Med Internet Res 2020;22(3):e16987. https://doi.org/10.2196/preprints.16987 PMid:32149711

14. The Sonosim Ultrasound Training Solution. Available from: https:// www.sonosim.com/ultrasound-training/?campaignid=9919140 382\&adgroupid=99948929265\&keyword=sonosim\&matchtype =e\&gclid=CjOKCQjwrlf3BRD1ARIsAMuugNtlh7gShZSk0gTrT 4nx66MZOUzHmK_j7FHI2Y0A3J3_3Shal5BxL4aAg4CEALw_ wcB. [Last accessed on 2020 Jun 11].

15. Apiratwarakul $K$, Pumiyoch $P$, lenghong $K$, Phungoen $P$, Gaysonsiri D, Bhudhisawasdi V. Endotracheal intubation on a stationary vs. moving ambulance. J Med Assoc Thai. 2020;103(6):18-21

16. lenghong K, Ussahgij W, Kanthachat K, Apiratwarakul K, Phungoen $\mathrm{P}$, Bhudhisawasdi V. Factors associated with severe intracranial pathology in acute non-traumatic headache patients in the emergency department. J Med Assoc Thai. 2020;103(6):47-50.

17. Apiratwarakul $\mathrm{K}$, lenghong $\mathrm{K}$, Gaysonsiri $\mathrm{D}$, Mitsungnern $\mathrm{T}$, Buranasakda $M$, Bhudhisawasdi $V$. The effectiveness of oxygen-powered inhalation devices in prehospital care. J Med Assoc Thai. 2020;103(6):58-60.

18. lenghong K, Kleebbuakwan K, Apiratwarakul K, Phungoen P, Gaysonsiri D, Bhudhisawasdi V. Comparison of cleaning methods for ultrasound probes at an emergency department in a resource-limited country. J Med Assoc Thai. 2020;103(6):67-71.

19. Apiratwarakul $K$, Songserm $W$, lenghong $K$, Phungoen $P$, Gaysonsiri D, Bhudhisawasdi V. The role of mechanical cardiopulmonary resuscitation devices in emergency medical services. J Med Assoc Thai. 2020;103(6):98-101. 